\title{
Inverter-based Voltage Control of Distribution Networks: A Three-Level Coordinated Method and Power Hardware-in-the-Loop Validation
}

\author{
Yu Wang, Member, IEEE, Mazheruddin H. Syed, Student Member, IEEE, Efren Guillo-Sansano, \\ Student Member, IEEE, Yan Xu, Senior Member, IEEE and Graeme M. Burt, Senior Member, IEEE
}

\begin{abstract}
The reactive power of the photovoltaic (PV) inverters has great potential for voltage regulation of distribution networks. In this paper, a new three-level coordinated control method for PV inverters is proposed to address network voltage fluctuation and violation issues. In Level I, a ramp-rate control is designed to smooth the network voltage fluctuations, while in Level II, a droop control is designed to alleviate the network voltage deviations. If the local compensation provided by Level I and II is not enough to regulate the network voltages within the required limits, the Level III control based on dynamic average consensus can respond and share the reactive power requirement among other inverters in a distributed way. The proposed control method can smooth the voltage profiles, restrain the voltage rise/drop problem, and coordinate all PV inverters in real-time when there is no feasible local solution. The stability analysis of the proposed three-level coordinated control for network voltage regulation is provided. The power hardware-in-the-loop (PHIL) experiment has been conducted for validating the proposed control method under various scenarios.
\end{abstract}

Index Terms - Voltage regulation, PV inverters, distributed control, power hardware-in-the-loop, distribution networks.

\section{INTRODUCTION}

$\mathrm{T}^{\mathrm{H}}$ HE penetration rate of power inverter-interfaced distributed energy resources (DERs) has been increasing largely in recent years. Among all kinds of DERs, rooftop photovoltaics (PVs) and plug-in electric vehicles (PEVs) are two major DERs in residential distribution networks [1], [2]. The reverse power flow caused by high PV generation can lead to voltage rise or over-voltage in the daytime [3]. While in the evening, the massive charging of PEVs increases residential load demand, which can result in voltage drop or under-voltage issues [4]. In addition, sudden voltage fluctuations can be caused by fast and stochastic power variation of PVs and PEVs. In some practices [5], the voltage fluctuations lasting ten seconds or longer at customer side should be $\pm 3 \%$ or less, regardless of the fluctuation frequency.

In power distribution networks, the bus voltages are conventionally regulated by mechanical devices such as on-

This work was supported The work in this paper was supported in part by Ministry of Education (MOE), Republic of Singapore, under grant AcRF TIER 1 2019-T1-001-069 (RG75/19), in part by the European Liaison on Electricity Committed Towards long-term Research Activity (ELECTRA) Integrated Research Program (IRP) and in part by the ERIGrid program. Y. Xu's work is also supported by Nanyang Assistant Professorship from Nanyang Technological University, Singapore. load tap changers, step voltage regulators and switched capacitors, etc. [6], [7]. However, such devices have a much lower response speed than the fast PV output change, and their lifetime will be reduced dramatically due to frequent tap/switch changing. In such circumstances, the IEEE 1547.8 working groups have been revising the standards to allow the inverters to contribute to voltage regulation in distribution networks [8].

The voltage control problem in power distribution networks are handled hierarchically in multi timescales by various devices. In long time-scales, the voltage regulation is usually formulated as an optimization problem [9], [10]. Since such methods require full information of the network and greater computation and communication capability, they are usually operated in larger dispatch intervals (e.g. 15mins). This paper focus on the real-time voltage control, which can be simply achieved in a decentralized manner based only on local voltage measurement. The decentralized voltage control by DER inverters can be further divided into $Q / P$ and $Q / V$ strategies [11], [12]. In $Q / P$ strategy, the reactive power output of the inverter is a function of its real power output, known as ramp-rate control [11]. In $Q / V$ strategy, the reactive power output of the inverter is dependent on the bus voltage, such as voltage droop control [12]. However, with the high uncertainty and plug-andplay ability of DERs, the system conditions can change rapidly. This makes the decentralized control alone difficult to handle the voltage regulation problem [13]. The network voltages may still violate the operation limits even with the specified droop settings and dispatched set-points. This makes real-time coordination among local controllers necessary.

To achieve real-time coordination among the multiple devices, distributed controls have become a promising solution, especially for sparse communication infrastructure as it is the case of distribution networks. The distributed control structure has the features of better cyber resilience and scalability compared to the centralized solution [14]. The distributed control has drawn increasing attention in the power research area [15]-[19]. In the earlier stage, distributed control based on leader-follower consensus algorithm is applied for network

Y. Wang and Y. Xu are with School of Electrical and Electronic Engineering, Nanyang Technological University, Singapore. (wangyulz@hotmail.com, eeyanxu@gmail.com).

M. .H. Syed, E. Guillo-Sansano and G. Burt are with Institute for Energy and Environment, University of Strathclyde, UK. (mazheruddin.syed@strath.ac.uk, efren.guillo-sansano@strath.ac.uk, graeme.burt@ strath.ac.uk). 
voltage regulation. In [15], the distributed cooperative control of multiple PV inverters is introduced. In [16], a distributed control for a group of $\mathrm{PV}$-storage systems has been proposed for network voltage rise issues. The methods in these works rely on the measurement and calculation from the leader controller. The distributed dynamic average consensus algorithm has been firstly applied for power system frequency control [17]. In contrast to the leader-follower consensus, the distributed average consensus calculates the average value of all units states in a fully distributed manner. Then this method has been applied for secondary voltage and frequency control in islanded microgrids [18], [19].

To increase the control system resilience, the local voltage control is still important, especially when the communication based distributed control cannot be performed. This inherently forms a hierarchical or multi-level voltage control scheme. In [13], a two-stage control which contains local droop control and distributed control is proposed. The voltage control scheme in [16] also contains local control and distributed control. In [20], a two-level voltage control by electrical springs is proposed, which contains both optimized dispatch and distributed operation. In [21], a voltage regulation scheme combined local and centralized control is proposed. In [22], the decentralized and distributed voltage control scheme is proposed. From the literature, droop control based methods [11], [12], leaderfollower consensus control [15], [16], and two-level control [20]-[22] have been proposed for network voltage regulation. It can be found that: (i) The real-time voltage flucations and voltage limit violations are not simultaneously considered. (ii) The distributed voltage regulation scheme relies on a leader (not fully distributed). (iii) The stability problems of multi-level coordinated voltage control are not systematically addressed. (iv) The distributed voltage control algorithms lack experimental validation to prove their real-world applicability.

Aiming to fill the above gaps, this paper proposes a new three-level coordinated voltage control approach that utilizes reactive power from PV inverters to deal with both voltage fluctuations and limits violations in distribution networks. The proposed control scheme is implemented and validated on a power hardware-in-the-loop (PHIL) experimental platform. Compared with most existing works with only simulations, the test platform emulates a more realistic environment for the evaluation of the proposed control scheme. The contributions of this paper are summarized as follows:

First, a three-level fully distributed voltage control scheme (i.e. ramp-rate control, droop control, and distributed control) is proposed. The proposed three-level operates autonomously according to the network voltage conditions. The guidelines for control gain selection for each control level are discussed.

Second, the closed-loop system stability of the proposed three-level control for voltage regulation in distribution networks is analyzed. The system stable conditions under different voltage ranges are derived.

Third, the proposed three-level control is validated by means of a PHIL experiment, where a real hardware inverter, separate distributed controller design, and realistic communications are incorporated. The results demonstrate the performance of the proposed control under various operating conditions, which adds to the practical value of the proposed method.

This paper is organized as follows. In Section II, preliminaries including modeling of distribution networks, PV inverters, and communication networks are introduced. Next, a three-level coordinated voltage control is presented in Section III. In Section IV, the stability analysis of the proposed control scheme is provided. The PHIL experiment is conducted and the results are discussed in detail in Section V. Finally, Section VI draws the conclusion.

\section{PRELIMINARIES}

In this section, the preliminaries including the models of distribution networks, PV inverters, and communication networks are presented.

\section{A. Power Distribution Networks}

For a distribution network with $N+1$ buses denoted by $i=0$, $1, \ldots, N$, bus $i=0$ is defined as the slack bus whose voltage can be adjusted by a substation transformer. The remaining $N$ buses are considered to be PQ buses, injecting or absorbing real and reactive power. The relationship between real power $\Delta P$ and reactive power $\Delta Q$ injection with respect to bus voltage magnitude $\Delta V$ and angle $\Delta \theta$ can be obtained from the Jacobian matrix by solving the power flow equations [11], [13]:

$$
\left[\begin{array}{l}
\Delta P(t) \\
\Delta Q(t)
\end{array}\right]=\left[\begin{array}{ll}
J_{1} & J_{2} \\
J_{3} & J_{4}
\end{array}\right]\left[\begin{array}{l}
\Delta \theta(t) \\
\Delta V(t)
\end{array}\right]
$$

where $\Delta P=\operatorname{col}\left\{\Delta P_{1}, \Delta P_{2}, \ldots, \Delta P_{N}\right\}, \Delta Q=\operatorname{col}\left\{\Delta Q_{1}, \Delta Q_{2}, \ldots, \Delta Q_{N}\right\}$, $\Delta V=\operatorname{col}\left\{\Delta V_{1}, \Delta V_{2}, \ldots, \Delta V_{N}\right\}$, and $\Delta \theta=\operatorname{col}\left\{\Delta \theta_{1}, \Delta \theta_{2}, \ldots, \Delta \theta_{N}\right\}$. The elements of matrix $\boldsymbol{J}_{1}, \boldsymbol{J}_{2}, \boldsymbol{J}_{3}$, and $\boldsymbol{J}_{4}$ are as below

$$
J_{1, i j}=\left[\frac{\partial P_{i}}{\partial \theta_{j}}\right], J_{2, i j}=\left[\frac{\partial P_{i}}{\partial V_{j}}\right], J_{3, i j}=\left[\frac{\partial Q_{i}}{\partial \theta_{j}}\right], J_{4, i j}=\left[\frac{\partial Q_{i}}{\partial V_{j}}\right] .
$$

Assuming that $\boldsymbol{J}_{1}$ is invertible, the deviation in bus voltage magnitude can be derived from (1) that

$$
\begin{aligned}
& \Delta V(t)=\left(\boldsymbol{J}_{4}-\boldsymbol{J}_{3} \boldsymbol{J}_{1}^{-1} \boldsymbol{J}_{2}\right)^{-1}\left[\Delta Q(t)-\boldsymbol{J}_{3} \boldsymbol{J}_{1}^{-1} \Delta P(t)\right] \\
& =\boldsymbol{S}_{V Q} \Delta Q(t)+\boldsymbol{S}_{V P} \Delta P(t)
\end{aligned}
$$

where the voltage sensitivity matrices are derived as $\boldsymbol{S}_{V Q}=\left(\boldsymbol{J}_{4}-\boldsymbol{J}_{3} \boldsymbol{J}_{1}^{-1} \boldsymbol{J}_{2}\right)^{-1}, \boldsymbol{S}_{V P}=-\left(\boldsymbol{J}_{4}-\boldsymbol{J}_{3} \boldsymbol{J}_{1}^{-1} \boldsymbol{J}_{2}\right)^{-1} \boldsymbol{J}_{3} \boldsymbol{J}_{1}^{-1}$. It should be noted that although $S_{V Q}$ and $S_{V P}$ are functions of $t$, the variations of them respect to $t$ are relatively small for a wide range of operating conditions [13], [23]. Equation (2) gives a linearized representation of $\Delta Q$ and $\Delta V$ near the equilibrium point, which is used to investigate the stability analysis in Section IV. In distribution networks, bus voltages are affected by both real and reactive power changes due to the high resistance to reactance ratio $(\mathrm{R} / \mathrm{X})$. For simplicity, the PV systems and load demand are aggregated at each bus. The PV inverters are operated to provide the maximum power output. Therefore, $\Delta P$ from $P V$ inverters is just considered as a disturbance which is not controllable. To mitigate the impact of 
$\Delta P$ on system voltages, a control approach to effectively control $\Delta Q$ from $\mathrm{PV}$ inverters is investigated. In this paper, the positive sign refers to power absorption while the negative sign to power injection.

\section{B. PV Inverters}

In grid-connected distribution networks, PV inverters are controlled in current control mode, functioning as current sources. The real power output of each PV inverter will follow the given PV data, while the reactive power output is controlled by the approach proposed in this paper.

To enable PV inverters to provide enough reactive power even when rated real power is being produced, the capacity of PV inverters can be oversized [24]. The available reactive power capacity of a PV inverter can be represented as:

$$
\begin{aligned}
\bar{Q}_{i}(t) & =\sqrt{S_{i}^{2}-P_{i}(t)^{2}} \\
S_{i} & =\left(1+\alpha_{i}\right) \bar{P}_{i}
\end{aligned}
$$

where $\bar{Q}_{i}(t)$ is the reactive power capacity of $i^{\text {th }} \mathrm{PV}$ inverter when the real power output is $P_{i}(t) . S_{i}, \bar{P}_{i}$ are the total capacity and rated real power of $i^{\text {th }} \mathrm{PV}$ inverter respectively. $\alpha_{i}$ is the percentage by which $i^{\text {th }} \mathrm{PV}$ inverter can be oversized in the deployment stage [25].

\section{Communication Network}

The communication network of PV inverters in the proposed control scheme can be described by a graph as $\mathcal{G}=(\mathcal{V}, \mathcal{E}, \mathcal{A})$, where $\mathcal{V}=\left\{v_{1}, v_{2}, \ldots, v_{N}\right\}$ is a set of $N$ nodes, and $\mathcal{E}=\mathcal{V} \times \mathcal{V}$ is a set of edges. Each node corresponds to the communication agent of a PV inverter while the edges represent the communication links for data exchange. If communication links are bidirectional, $\left(v_{i}, v_{j}\right) \in \mathcal{E} \Rightarrow\left(v_{j}, v_{i}\right) \in \mathcal{E} \forall i, j$ the graph is said to be undirected, otherwise, it is directed. The adjacency matrix $\mathcal{A}=\left[a_{i j}\right]$ is associated with the edges, $a_{i j}$ represents the weight for information exchange between agents $i$ and $j$, where $a_{i j}=1$ if agents $i$ and $j$ are connected through an edge $\left(v_{i}, v_{j}\right) \in E$, otherwise, $a_{i j}=0$. The in-degree matrix is defined as $\boldsymbol{D}=\operatorname{diag}\left(d_{i}\right)$, where $d_{i}=\sum_{i=1}^{N} a_{i j}$. The Laplacian matrix is defined as $\boldsymbol{L}=\left[l_{i j}\right]$, where $l_{i j}$ is the element in $\boldsymbol{L}$, and $\boldsymbol{L}=\boldsymbol{D}-\mathcal{A}$. For a real-world implementation, communication can be achieved through both cable communications and wireless communications like WiFi or Zigbee technology.

\section{THREE-LEVEl COORDINATED VOLTAGE CONTROL}

The proposed control approach comprises three hierarchical levels to control the voltage at different ranges, as illustrated in Fig.1. The Level I ramp-rate control aims to smooth the voltage fluctuations and operates at all voltage ranges. The Level II droop control aims to mitigate voltage deviations when the bus voltage is out of the desirable range $[\underset{\sim}{V}, \tilde{V}]$. The Level III distributed control will be activated when the bus voltage is beyond the allowable range $[\underline{V}, \bar{V}]$. Note that the Level I and II controls are operated based on local voltage measurement only, while Level III control relies on the voltage signals from adjacent inverters via a sparse communication network.

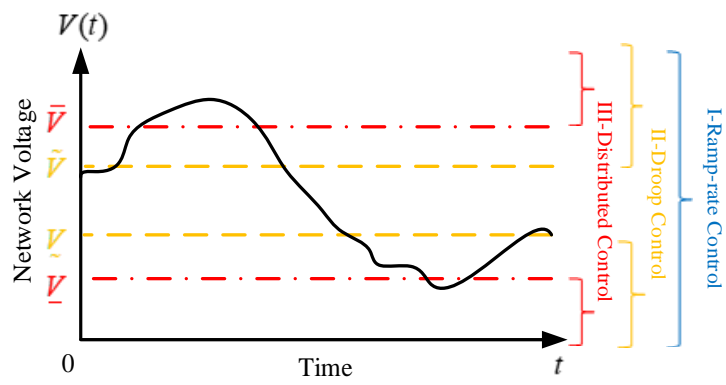

Fig. 1. The operational ranges of the proposed three-level control.

The objective of the proposed control approach is to determine an appropriate utilization ratio for each inverter. The utilization ratio $u_{i}(t)$ of $i^{\text {th }} \mathrm{PV}$ inverter is defined as the reactive power output $\Delta Q_{i}(t)$ divided by the reactive power capacity $\bar{Q}_{i}(t)$, i.e. $u_{i}(t)=\Delta Q_{i}(t) / \bar{Q}_{i}(t)$. Fig. 2 shows the overall architecture of the proposed control method. Each control level of the proposed control approach can calculate a utilization ratio, i.e. $u_{i}^{I}(t), u_{i}^{I I}(t)$, and $u_{i}^{I I I}(t)$, according to the network voltage conditions. The utilization ratio $u_{i}(t)$ of $i^{\text {th }} \mathrm{PV}$ inverter is the sum of these three levels' utilization ratios. The final reactive power output of $i^{\text {th }} \mathrm{PV}$ inverter is calculated from

$$
\left\{\begin{array}{l}
u_{i}(t)=u_{i}^{I}(t)+u_{i}^{I I}(t)+u_{i}^{I I I}(t) \\
\Delta Q_{i}(t)=u_{i}(t) \cdot \bar{Q}_{i}(t) \\
u_{i}(t), u_{i}^{I}(t), u_{i}^{I I}(t), u_{i}^{I I I}(t) \in[-1,1] .
\end{array}\right.
$$

The details of each individual control level are explained in the following sub-sections.

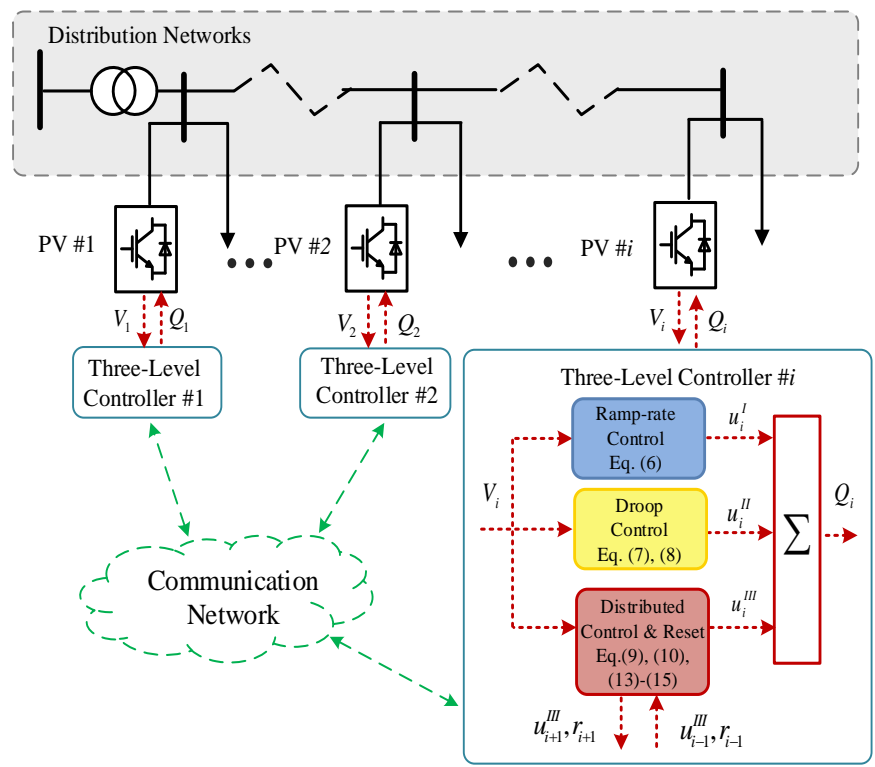

Fig. 2. The overall architecture of the proposed control scheme. 


\section{A. Level I: Ramp-rate Control}

The objective of Level I control is to locally regulate voltage ramp-rate within a limit, i.e. $\left|\frac{V_{i}\left(t_{1}\right)-V_{i}\left(t_{2}\right)}{t_{1}-t_{2}}\right| \leq V R R_{\lim }$, where $V R R_{\text {lim }}$ is the limit of voltage ramp-rate.

The moving clouds (shading effect) can lead to significant voltage fluctuations, which can be locally smoothed by appropriate reactive power injection [26]. A moving average filter based ramp-rate controller is designed for each PV inverter. The high-frequency components of each bus voltage are extracted by subtracting the filtered voltage from the measured voltage and compensated with the inverter reactive power output determined by ramp-rate control. The utilization ratio of Level I control is updated as:

$$
u_{i}^{I}(t)=K_{i}^{I}\left[V_{i}(t)-\frac{\sum_{j=t-\omega}^{t} V_{i}(j)}{T(t)-T(t-\omega)}\right]
$$

where $\omega>0$ is the length of the moving average window and $K_{i}^{I}>0$ is the ramp-rate control gain.

Remark 1 (Level I control parameters selection): Generally, increasing the moving average filter window length $\omega$ will lead to more low-frequency components being compensated. The ramp-rate control gain $K_{i}^{I}$ is proportional to the compensation effect of the ramp-rate control. The boundaries of control gain selection can be obtained from the stability analysis in Section IV. The criterion for selection of the ramp-rate control parameters in this paper is to guarantee that even under a large change of load demand, the network voltage fluctuations still satisfy the industrial requirement [5].

\section{B. Level II: Droop Control}

The objective of Level II control is to locally mitigate the network voltage deviations, i.e. $V_{i}^{\prime}(t) \rightarrow V_{i}(t)$, when the local bus voltage is out of the defined voltage range $[\underset{\sim}{V}, \tilde{V}]$. A droop control will be activated to generate/absorb reactive power proportional to the bus voltage deviation. The utilization ratio of Level II control is updated as:

$$
u_{i}^{I I}(t)=\left\{\begin{array}{l}
K_{i}^{I I}\left(V_{i}(t)-\tilde{V}\right), \quad V_{i}(t)>\tilde{V} \\
0, \quad \underset{\sim}{V} \leq V_{i}(t) \leq \tilde{V} \\
K_{i}^{I I}\left(V_{i}(t)-\underset{\sim}{V}\right), \quad V_{i}(t)<\underset{\sim}{V}
\end{array}\right.
$$

$K_{i}^{I I}>0$ is the droop gain of the $i^{\text {th }} \mathrm{PV}$ inverter. $[\underset{\sim}{V}, \tilde{V}]$ represents the dead band for the droop control.

Remark 2 (Level II control parameters selection): There are several methods to determine the voltage droop control parameters. A widely used method is to choose the droop gain proportional to the capacity of the inverters, where the line impedances are ignored [27]. Another method is adjusting droop coefficient adaptively to achieve a certain objective (e.g. economic dispatch and loss minimization) [28]. In distribution networks, to avoid the instability caused by improper droop settings, it is suggested to choose the droop settings following voltage sensitivity analysis, especially when the distribution network is large [13], [29].

In this paper, the droop control gain of each inverter is selected based on the voltage sensitivity analysis as follows:

$$
K_{i}^{I I}=\beta_{i} \frac{1}{s_{i i}}
$$

where $s_{i i}=\partial V_{i} / \partial Q_{i} \in S_{Q V}$ is obtained from (1) and (2), $\beta_{i}$ is a scaler of the droop control gain. The boundaries of control gain selection should follow the stability analysis in Section IV.

\section{Level III: Distributed Control}

In Level III of the proposed control, a distributed voltage control and its distributed reset algorithm to terminate the distributed controllers are proposed. The objective of Level III control is to coordinate all available PV inverters when network voltages cannot be regulated within $[\underline{V}, \bar{V}]$ locally, where the steady-state conditions can be represented as follows:

$$
\begin{cases}\lim _{t \rightarrow \infty}\left\|V_{i}(t)-\bar{V}\right\|=0, & V_{i}(t)>\bar{V} \\ \lim _{t \rightarrow \infty}\left\|V_{i}(t)-\underline{V}\right\|=0, & V_{i}(t)<\underline{V}\end{cases}
$$

In such circumstances, all PV inverters with available capacity will increase their reactive power output until all network voltages are back to the allowable range. This control will be deactivated when the network voltages are recovered within a safe range, which is achieved by a distributed reset algorithm proposed in this subsection.

The utilization ratio of Level III control is updated based on the following equations as:

$$
\begin{gathered}
\dot{u}_{i}^{I I I}(t)=G_{i}^{I I I}\left[\sum_{i=1}^{N} a_{i j}\left(u_{j}^{I I I}(t)-u_{i}^{I I I}(t)\right)\right]+e(t) \\
e(t)=\left\{\begin{array}{cc}
K_{i}^{I I I}\left(V_{i}(t)-\bar{V}\right), \quad V_{i}>\bar{V} \\
0, & \underline{V} \leq V_{i} \leq \bar{V} \\
K_{i}^{I I I}\left(V_{i}(t)-\underline{V}\right), \quad V_{i}<\underline{V}
\end{array}\right.
\end{gathered}
$$

where $K_{i}^{I I I}>0$ and $G_{i}^{I I I}>0$ are the distributed control gains.

The proposed distributed control reaches consensus (i.e. steady-state), for any initial state, when the following conditions are met:

$$
\lim _{t \rightarrow \infty}\|e(t)\|=0, \lim _{t \rightarrow \infty}\left\|u_{i}^{I I I}(t)-u_{j}^{I I I}(t)\right\|=0
$$

Equation (12) ensures the control objectives in (9) are met. The proposed control can guarantee that the voltage deviations out of $[\underline{V}, \bar{V}]$ are eliminated in steady-state with the utilization ratios of Level III control for each inverter equalized.

Remark 3 (Level III control gain selection): $K_{i}^{I I I}$ and $G_{i}^{I I I}$ determines the convergence speed of the proposed distributed control. Generally, the larger $K_{i}^{I I I}$ and $G_{i}^{I I I}$ are chosen, the faster the convergence speed will be. However, the trade-off between robustness and convergence speed should also be 
considered when choosing $K_{i}^{I I I}$ and $G_{i}^{I I I}$. The stability criteria for Level III control gain selection is further discussed in Section IV .

Furthermore, integrators in (9) should be reset when all network bus voltages are back to the safe operating range. In order to be consistent with the ideology of distributed control, the integrators also need to be reset in a fully distributed manner This motivates us to design a distributed reset algorithm for the proposed distributed control. To this end, a flag signal of each inverter is defined as:

$$
F_{i}(t)=\left\{\begin{array}{c}
0, \frac{V+\underline{V}}{2} \leq V_{i}(t) \leq \frac{\tilde{V}+\bar{V}}{2} \\
1, \quad \text { otherwise }
\end{array}\right.
$$

where $(\tilde{V}+\bar{V}) / 2$ and $(\underset{\sim}{V}+\underline{V}) / 2$ are the upper and lower bound to trigger the flag value, respectively. The flag signal will turn from 0 to 1 when the voltage is out of the range defined in (13). The reset signal $R_{i}(t)$ of each inverter will be updated according to the following equations:

$$
\begin{gathered}
\dot{r}_{i}(t)=\chi_{i} \sum_{i=1}^{N} a_{i j}\left(r_{j}(t-\tau(t))-r_{i}(t)\right)+\gamma_{i}\left(F_{i}(t)-r_{i}(t)\right) \\
R_{i}(t)=\operatorname{sign}\left[r_{i}(t)\right]
\end{gathered}
$$

where $r_{i}(t)$ and $R_{i}(t)$ are the transmitted signal and the reset signal of the $i^{\text {th }}$ inverter, respectively. $\chi_{i}>0$ and $\gamma_{i}>0$ are two coefficients which influence the convergence speed of the algorithm. It should be noted that $r_{i}(t)$ shares the same communication network as of (9). The integrators for the distributed control will be reset when $R_{i}(t)$ falls from 1 to 0 . According to the distributed reset algorithm (13)-(15), $\forall V_{i}(t) \notin\left[\left({ }_{\sim}^{V}+\underline{V}\right) / 2,(\tilde{V}+\bar{V}) / 2\right] \Rightarrow \forall F_{i}(t), R_{i}(t) \neq 0 \quad$, which ensures the integrators can be reset in a distributed manner when the voltages return to the defined voltage range in (13).

\section{STABILITY ANALYSIS}

In order to analyze the closed-loop system stability under the proposed control, we can rewrite the proposed three-level control (5) as:

$$
\Delta Q_{i}=u_{i} \cdot \bar{Q}_{i}+\bar{Q}_{i}^{d}
$$

It should be noted that $\bar{Q}_{i}^{d}=0$ when there is no PV generation. During the daytime, the reactive power from PV inverter contains both controllable term $u_{i} \cdot \bar{Q}_{i}$ and a disturbance term $\bar{Q}_{i}^{d} \cdot \bar{Q}_{i}$ is a constant indicating the available reactive power capacity of PV inverter for a certain period. In this section, the symbol of time $t$ is omitted for brevity.

In matrix form, (16) can be written as:

$$
\Delta Q=\bar{Q} u+\bar{Q}^{d}
$$

where vectors $u=\operatorname{col}\left\{u_{1}, u_{2}, \ldots, u_{N}\right\}, \bar{Q}^{d}=\operatorname{col}\left\{\bar{Q}_{1}^{d}, \bar{Q}_{2}^{d}, \ldots, \bar{Q}_{N}^{d}\right\}$, and matrix $\overline{\boldsymbol{Q}}=\operatorname{diag}\left\{\bar{Q}_{1}, \bar{Q}_{2}, \ldots, \bar{Q}_{N}\right\}$.
Combing (2) and (17), the system can be rearranged in matrix form as:

$$
\begin{aligned}
& \Delta V=\boldsymbol{S}_{V Q} \overline{\boldsymbol{Q}}_{u}+\boldsymbol{S}_{V Q} \bar{Q}^{d}+\boldsymbol{S}_{V P} \Delta P \\
& =\boldsymbol{S}_{V Q} \overline{\boldsymbol{Q}} u+\boldsymbol{W}
\end{aligned}
$$

where $\boldsymbol{W}=\boldsymbol{S}_{V Q} \overline{\boldsymbol{Q}}^{d}+\boldsymbol{S}_{V P} \Delta \boldsymbol{P}$ is the uncontrollable term, which can be viewed as a disturbance. The system under the proposed control can be derived into three operational ranges, which is illustrated in Fig. 1.

\section{A. Level I Control for $V_{i} \in[\underset{\sim}{V}, \tilde{V}]$}

In this operational range, only the ramp-rate control will take effect. The ramp-rate control functions like derivative control, which generates a control signal $u_{i}^{I}$ based on the derivate of voltage $(\mathrm{dV} / \mathrm{dt})$. As the ramp-rate control based on moving average filter is not a linear controller, the approximation dynamics can be obtained from:

$$
u_{i}=K_{i}^{I} \frac{s}{s+\hat{\omega}} V_{i}
$$

where $\hat{\omega}_{i}$ is a parameter corresponding to the window length of the moving average filter.

Substituting (19) into (18), we can obtain the system dynamics in matrix form as:

$$
\begin{aligned}
& \Delta \dot{V}+\hat{\boldsymbol{\omega}} \Delta V=\overline{\boldsymbol{Q}} \boldsymbol{S}_{V Q} \boldsymbol{K}^{I} \Delta \dot{V}+\dot{\boldsymbol{W}}+\hat{\boldsymbol{\omega}} \boldsymbol{W} \\
& \text { i.e. } \Delta \dot{V}=-\left(\boldsymbol{I}-\overline{\boldsymbol{Q}} \boldsymbol{S}_{V Q} \boldsymbol{K}^{I}\right) \hat{\boldsymbol{\omega}} \Delta V+\boldsymbol{W}^{I}
\end{aligned}
$$

where $\hat{\boldsymbol{\omega}}=\operatorname{diag}\left\{\hat{\omega}_{1}, \hat{\omega}_{2}, \ldots, \hat{\omega}_{N}\right\}, \boldsymbol{K}^{I}=\operatorname{diag}\left\{K_{1}^{I}, K_{2}^{I}, \ldots, K_{N}^{I}\right\}$. $\boldsymbol{W}^{I}=\dot{\boldsymbol{W}}+\hat{\boldsymbol{\omega}} \boldsymbol{W}, \boldsymbol{I}$ is an identity matrix of the appropriate dimension.

If the system is stable, the system matrix in (21) should be Hurwitz, i.e. $-\left(\boldsymbol{I}-\overline{\boldsymbol{Q}} \boldsymbol{S}_{V Q} \boldsymbol{K}^{I}\right) \hat{\boldsymbol{\omega}} \prec 0$. As $\hat{\boldsymbol{\omega}}$ of the is positive definite, thus it requires $\boldsymbol{I}-\overline{\boldsymbol{Q}} \boldsymbol{S}_{V Q} \boldsymbol{K}^{I} \succ 0$. Therefore, the chosen value of $\boldsymbol{K}^{I}$ should satisfy all the eigenvalues $\operatorname{Re}\left[\lambda_{i}\left(\boldsymbol{I}-\overline{\boldsymbol{Q}} \boldsymbol{S}_{V Q} \boldsymbol{K}^{I}\right)\right]>0$.

\section{B. Two-Level Control for $V_{i} \in[\underline{V}, \underset{\sim}{V}] \cup[\tilde{V}, \bar{V}]$}

In this operational range, both ramp-rate control and droop control will take effect. The droop control in (7) can be rewritten as:

$$
\begin{gathered}
u_{i}^{I I}=K_{i}^{I I}\left[\left(V_{i}-V^{*}\right)-\left(V_{i}^{I I}-V^{*}\right)\right]=K_{i}^{I I}\left(\Delta V_{i}-\Delta V_{i}^{I I}\right) \\
\text { where } V_{i}^{I I}=\left\{\begin{array}{cc}
\tilde{V}, & V_{i}(t)>\tilde{V} \\
V_{i}, \underset{\sim}{V} \leq V_{i}(t) \leq \tilde{V} \\
\underset{\sim}{V}, & V_{i}(t)<\underset{\sim}{V}
\end{array}\right.
\end{gathered}
$$

and $V^{*}$ is the equilibrium points of $\Delta V$ in the system model, which is set as 1 p.u. in this paper. 
Substituting the control law of (19) and (22) into (18), and rearranging in matrix form we get:

$$
\begin{aligned}
& \Delta \dot{V}+\hat{\boldsymbol{\omega}} \Delta V=\overline{\boldsymbol{Q}} S_{V Q}\left(\boldsymbol{K}^{I} \Delta \dot{V}+\boldsymbol{K}^{I I} \Delta \dot{V}+\right. \\
& \left.\boldsymbol{K}^{I I} \hat{\boldsymbol{\omega}}\left(\Delta V+\Delta V^{I I}\right)\right)+\dot{\boldsymbol{W}}+\hat{\boldsymbol{\omega}} \boldsymbol{W}
\end{aligned}
$$

also representable as

$$
\Delta \dot{V}=\left[\boldsymbol{I}-\overline{\boldsymbol{Q}} \boldsymbol{S}_{V Q}\left(\boldsymbol{K}^{I}+\boldsymbol{K}^{I I}\right)\right]^{-1}\left(\overline{\boldsymbol{Q}} \boldsymbol{S}_{V Q} \boldsymbol{K}^{I I}-\boldsymbol{I}\right) \hat{\boldsymbol{\omega}} \Delta V+\boldsymbol{W}^{I I}
$$

where $\Delta V^{I I}=\operatorname{col}\left\{\Delta V_{1}^{I I}, \Delta V_{2}^{I I}, \ldots, \Delta V_{N}^{I I}\right\}, \boldsymbol{K}^{I I}=\operatorname{diag}\left\{K_{1}^{I I}, K_{2}^{I I}, \ldots, K_{N}^{I I}\right\}$, $\boldsymbol{W}^{I I}$ is the disturbance term which can be derived from above equations.

Therefore, for the dynamic system of (24), the system stability requires the following matrix to be Hurwitz:

$$
\boldsymbol{\Psi}=\left[\boldsymbol{I}-\overline{\boldsymbol{Q}} \boldsymbol{S}_{V Q}\left(\boldsymbol{K}^{I}+\boldsymbol{K}^{I I}\right)\right]^{-1}\left(\overline{\boldsymbol{Q}} \boldsymbol{S}_{V Q} \boldsymbol{K}^{I I}-\boldsymbol{I}\right) \hat{\boldsymbol{\omega}} \prec 0
$$

For a certain control gain $\boldsymbol{K}^{I}$ in Level I control, the chosen value of $\boldsymbol{K}^{I I}$ should satisfy all the eigenvalues $\operatorname{Re}\left[\lambda_{i}(\Psi)\right]<0$.

\section{Three-Level Control for $V_{i} \notin[\underline{V}, \bar{V}]$}

In this operational range, all three control levels take effect. In matrix form, the distributed controller in level III can be represented as:

$$
\begin{gathered}
\dot{u}^{I I I}=-\boldsymbol{G}^{I I I} \boldsymbol{L} u^{I I I}+\boldsymbol{e}=-\boldsymbol{G}^{I I I} \boldsymbol{L} u^{I I I}+\boldsymbol{K}^{I I I}\left(V-V^{I I I}\right) \\
=-\boldsymbol{G}^{I I I} \boldsymbol{L} u^{I I I}+\boldsymbol{K}^{I I I}\left[\left(V-V^{*}\right)-\left(V^{I I I}-V^{*}\right)\right] \\
=-\boldsymbol{G}^{I I I} \boldsymbol{L} u^{I I I}+\boldsymbol{K}^{I I I}\left(\Delta V-\Delta V^{I I I}\right) \\
\text { where } V_{i}^{I I I}=\left\{\begin{array}{cc}
\bar{V}, & V_{i}(t)>\bar{V} \\
V_{i}, \underline{V} \leq V_{i}(t) \leq \bar{V} \\
\underline{V}, & V_{i}(t)<\underline{V}
\end{array}\right.
\end{gathered}
$$

Substituting all three level control laws into (18), we obtain:

$\Delta \dot{V}=\left[\boldsymbol{I}-\overline{\boldsymbol{Q}} \boldsymbol{S}_{V Q}\left(\boldsymbol{K}^{I}+\boldsymbol{K}^{I I}\right)\right]^{-1}\left[\left(\overline{\boldsymbol{Q}} \boldsymbol{S}_{V Q} \boldsymbol{K}^{I I}-\boldsymbol{I}\right) \hat{\boldsymbol{\omega}}+\overline{\boldsymbol{Q}} \boldsymbol{S}_{V Q} \boldsymbol{K}^{I I I}\right] \Delta V$ $+\left[\boldsymbol{I}-\overline{\boldsymbol{Q}} \boldsymbol{S}_{V Q}\left(\boldsymbol{K}^{I}+\boldsymbol{K}^{I I}\right)\right]^{-1}\left[\overline{\boldsymbol{Q}} \boldsymbol{S}_{V Q}\left(-\boldsymbol{G}^{I I I} \boldsymbol{L}+\hat{\boldsymbol{\omega}}\right) u^{I I I}\right]+\boldsymbol{W}^{I I I}$

where $\boldsymbol{K}^{I I I}=\operatorname{diag}\left\{K_{1}^{I I I}, K_{2}^{I I I}, \ldots, K_{N}^{I I I}\right\}, \boldsymbol{G}^{I I I}=\operatorname{diag}\left\{G_{1}^{I I I}, G_{2}^{I I I}, \ldots, G_{N}^{I I I}\right\}$, $W^{I I I}$ is the disturbance term.

Combining (26) and(27) into the state-space form, one can obtain:

$$
\left[\begin{array}{c}
\Delta \dot{V} \\
\dot{u}^{I I I}
\end{array}\right]=\left[\begin{array}{cc}
\Gamma & \Xi \\
\boldsymbol{K}^{I I I} & -\boldsymbol{G}^{I I I} \boldsymbol{L}
\end{array}\right]\left[\begin{array}{l}
\Delta V \\
u^{I I I}
\end{array}\right]+\left[\begin{array}{c}
\boldsymbol{W}^{I I I} \\
-\boldsymbol{K}^{I I I}\left(V^{I I I}-V^{*}\right)
\end{array}\right]
$$

where

$$
\begin{gathered}
\Gamma=\left[\boldsymbol{I}-\overline{\boldsymbol{Q}} \boldsymbol{S}_{V Q}\left(\boldsymbol{K}^{I}+\boldsymbol{K}^{I I}\right)\right]^{-1}\left[\left(\overline{\boldsymbol{Q}} \boldsymbol{S}_{V Q} \boldsymbol{K}^{I I}-\boldsymbol{I}\right) \hat{\boldsymbol{\omega}}+\overline{\boldsymbol{Q}} \boldsymbol{S}_{V Q} \boldsymbol{K}^{I I I}\right] \\
\boldsymbol{\Xi}=\left[\boldsymbol{I}-\overline{\boldsymbol{Q}} \boldsymbol{S}_{V Q}\left(\boldsymbol{K}^{I}+\boldsymbol{K}^{I I}\right)\right]^{-1}\left[\overline{\boldsymbol{Q}} \boldsymbol{S}_{V Q}\left(-\boldsymbol{G}^{I I I} \boldsymbol{L}+\hat{\boldsymbol{\omega}}\right)\right]
\end{gathered}
$$

The system stability requires the following matrix to be Hurwitz:

$$
\left[\begin{array}{cc}
\Gamma & \boldsymbol{\Xi} \\
\boldsymbol{K}^{I I I} & -\boldsymbol{G}^{I I I} \boldsymbol{L}
\end{array}\right] \prec 0
$$

Therefore, based on the derived closed-loop systems in (21), (25) and (29), the trace of eigenvalues can be ploted to exam the stability boundaries of the studied system.

\section{PHIL EXPERIMENTAL TESTS}

\section{A. Test System}

In this section, the proposed three-level coordinated voltage control method is validated in a 7-bus distribution network [13] through a power hardware-in-the-loop test. The single line diagram of the test system is shown in Fig. 3 and the PV capacity and load demand at each bus are modified as in Table I. One-way communication among PV inverters are considered, which forms a directed circuit communication graph as shown in Fig. 3. As all the PV inverters are connected by a directed communication graph, the convergence of the proposed distributed control is guaranteed. The tap change of the OLTC is not considered and the voltage at bus 0 is kept at 1.0 p.u. during the operation. Based on national standards of different countries, distribution networks are usually allowed a maximum voltage deviation of $\pm 5 \%$ - $\pm 10 \%$ [30]. Therefore, in this paper, the voltage ranges to trigger distributed control and droop control are chosen as $[\underline{V}, \bar{V}]=[0.95,1.05]$ and $[\underset{\sim}{V}, \tilde{V}]=[0.97,1.03]$.

In order to test the proposed control algorithm in a more realistic environment, a PHIL experiment is conducted [31]. Fig. 4 shows the real-time power hardware-in-the-loop test platform utilized in this paper. The PV inverter and load demand at bus 5 are replaced with real laboratory equipment. A $15 \mathrm{kVA}$ inverter and a $10 \mathrm{~kW}$ resistive load bank with a $90 \mathrm{kVA}$ interfacing converter are used, as shown in Fig. 4. The 90kVA interfacing converter reproduces the voltages from the simulation point of common coupling within the laboratory while at the same time measures the hardware response (currents) to be fed in at the simulation point of common coupling. The voltage and current signals are scaled up and down between the real-time simulation and the hardware equipment. To increase the accuracy of the PHIL implementation, time delay compensation technique presented in [32] has been used. The remainder of the 7-bus distribution network is simulated in a digitial real-time sumlator (RTDS). It should be noted that the inner voltage and current control loop dynamics for the hardware PV inverter are considered as in [33], while remaining six inverters are simplified into controlled current sources.

The proposed control scheme is embedded in a separate instance for each of the PV inverters within the RTDS. The distribution network model in RTDS is simulated at a time step of $50 \mu \mathrm{s}$. The network voltages are sampled with a slower rate of $10 \mathrm{~ms}$, which is acquired by the proposed three-level controllers to generate control signals. Furthermore, all the 
communications required for the distributed control algorithm are implemented through a real communication network. The control signal from each of the dedicated controller instance is independently routed outside of the RTDS using GTNET cards employing user datagram protocol (UDP) into a communication emulator, and back into neighboring controller instance in the RTDS. Such an experiment setup represents close to real-world implementation allowing for the feasibility of the proposed approach to be validated.

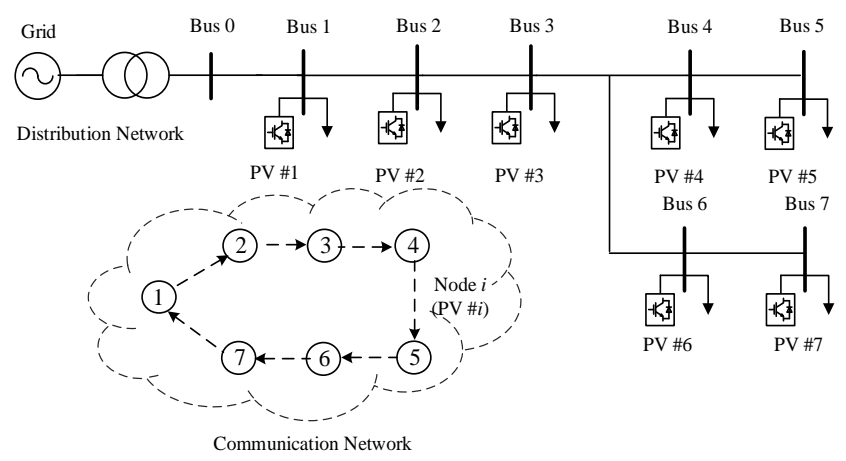

Fig. 3. The configuration of the 7-bus distribution network and the communication graph of PV inverters.

TABLE I

THE PV CAPACITY AND LOAD DEMAND IN THE 7-BUS NETWORK

\begin{tabular}{c|c|c}
\hline \hline Bus ID & PV Capacity (kVA) & Load Demand (kVA) \\
\hline Bus 1 & 850 & $700+100 \mathrm{j}$ \\
\hline Bus 2 & 600 & $850+250 \mathrm{j}$ \\
\hline Bus 3 & 1200 & $600+150 \mathrm{j}$ \\
\hline Bus 4 & 900 & $1250+500 \mathrm{j}$ \\
\hline Bus 5 & 800 & $900+300 \mathrm{j}$ \\
\hline Bus 6 & 900 & $100+100 \mathrm{j}$ \\
\hline Bus 7 & 1000 & $1000+350 \mathrm{j}$ \\
\hline \hline
\end{tabular}

PARAMETERS OF PROPOSED THREE-LEVEl CONTROL

\begin{tabular}{c|c}
\hline \hline Level I & $K_{i}^{I}=15$ and $\omega_{i}=10$ \\
\hline Level II & $K_{i}^{I I}=10$ \\
\hline Level III & $K_{i}^{I I I}=1, G_{i}^{I I I}=1, \chi_{i}=2, \gamma_{i}=1$ \\
\hline
\end{tabular}

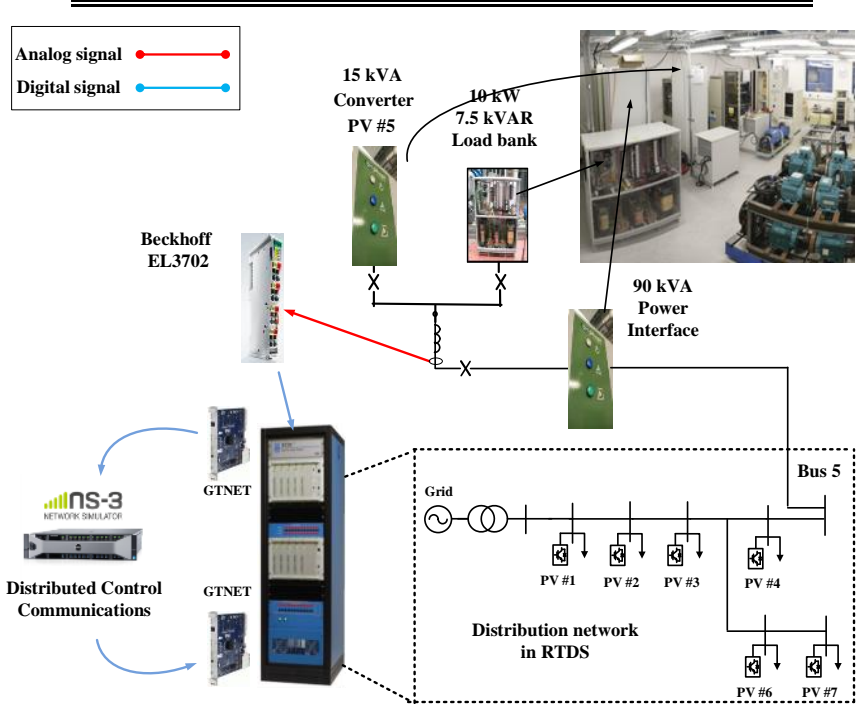

Fig. 4. Power hardware-in-the-loop test platform.

\section{B. Eigenvalue Analysis}

The stability analysis in Section IV is applied to the 7-bus distribution network in this section, with the control parameters as given in Table II. The three-level control for $V_{i} \notin[\underline{V}, \bar{V}]$ in Section IV-C is studied as an example. The trace of eigenvalues as control gains $K_{i}^{I I I}$ and $G_{i}^{I I I}$ varying are shown in Fig. 5 and Fig. 6. Arrows indicate the direction of increasing gain. Significant negative eigenvalues are omitted for clarity. It can be observed that increasing the control gains in Level III leads the system to instability. In Fig. $5, G_{i}^{I I I}=1$ keeps unchange and eigenvalue traces cross the negative half plane of real axis when $K_{i}^{I I I}>35$, which indicates system unstable. Similarly, in Fig. $6, K_{i}^{I I I}=1$ keeps unchanged and the eigenvalue traces cross the negative half plane of real axis when $G_{i}^{I I I}>70$. Generally, increasing of control gains $K_{i}^{I I I}$ and $G_{i}^{I I I}$ will speed up the convergence but could lead to system oscillation and instability.

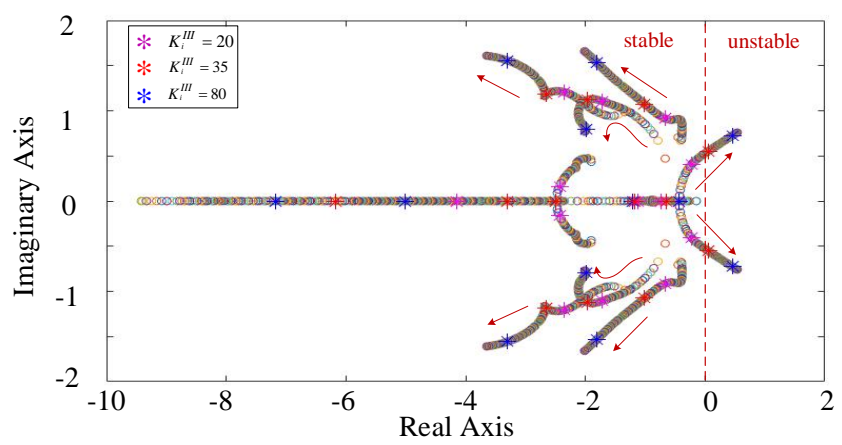

Fig. 5. The eigenvalue traces of closed-loop system (28) as controller gains $G_{i}^{I I I}=1, K_{i}^{I I I}$ varying from $[1,100]$.

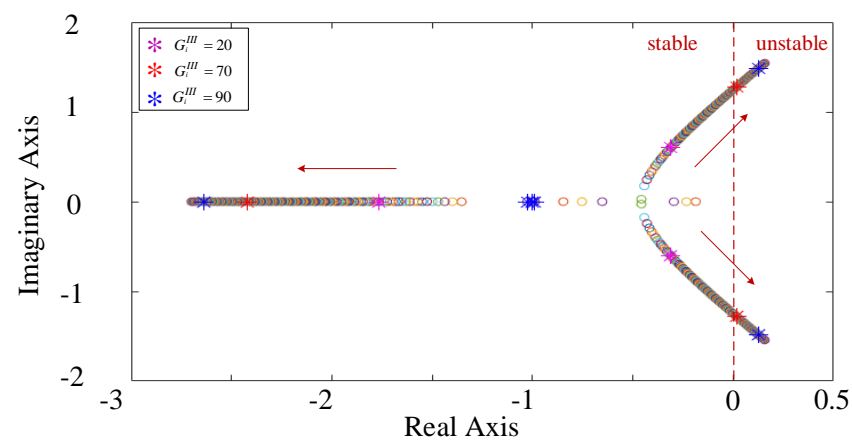

Fig. 6. The eigenvalue traces of closed-loop system (28) as controller gains $K_{i}^{I I I}=1, G_{i}^{I I I}$ varying from $[1,100]$.

\section{Test Case 1: Voltage Drop with Step Load Change}

In Test Case 1, the effectiveness of the proposed control approach to mitigate sudden voltage drops due to step changes in load is tested. Such a situation would normally occur in the evening in distribution grids when load demand is high but there is a lack of PV generation. As there is no PV production, all capacity of PV inverters can be utilized to supply reactive 
power for network voltage regulation. The communication delay in the distributed control is not considered in Test Case 1. Three step changes in load are emulated, in terms of percentage representable as: (i) an increase from $35 \%$ to $65 \%$ at $t=30 \mathrm{~s}$; (ii) a further increase from $65 \%$ to $100 \%$ at $t=130 \mathrm{~s}$; and (iii) a decrease from $100 \%$ to $35 \%$ at $t=230 \mathrm{~s}$.

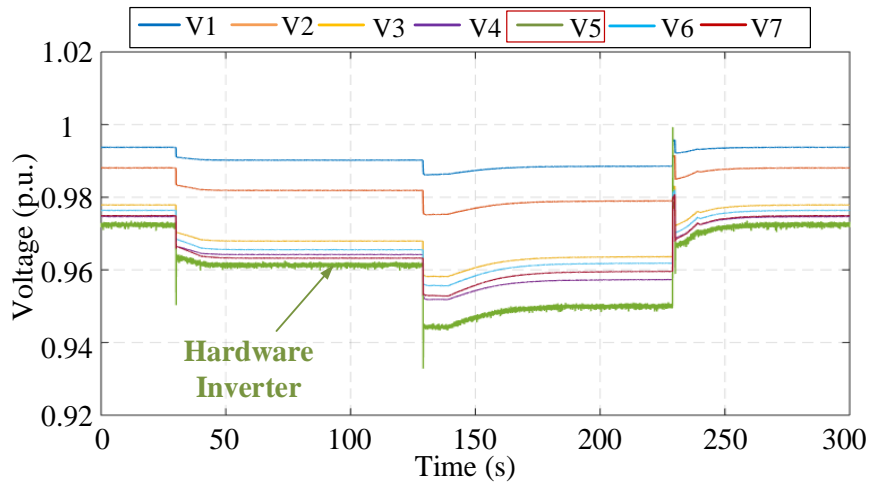

Fig. 7. Voltage profiles of the distribution network in Test Case 1.
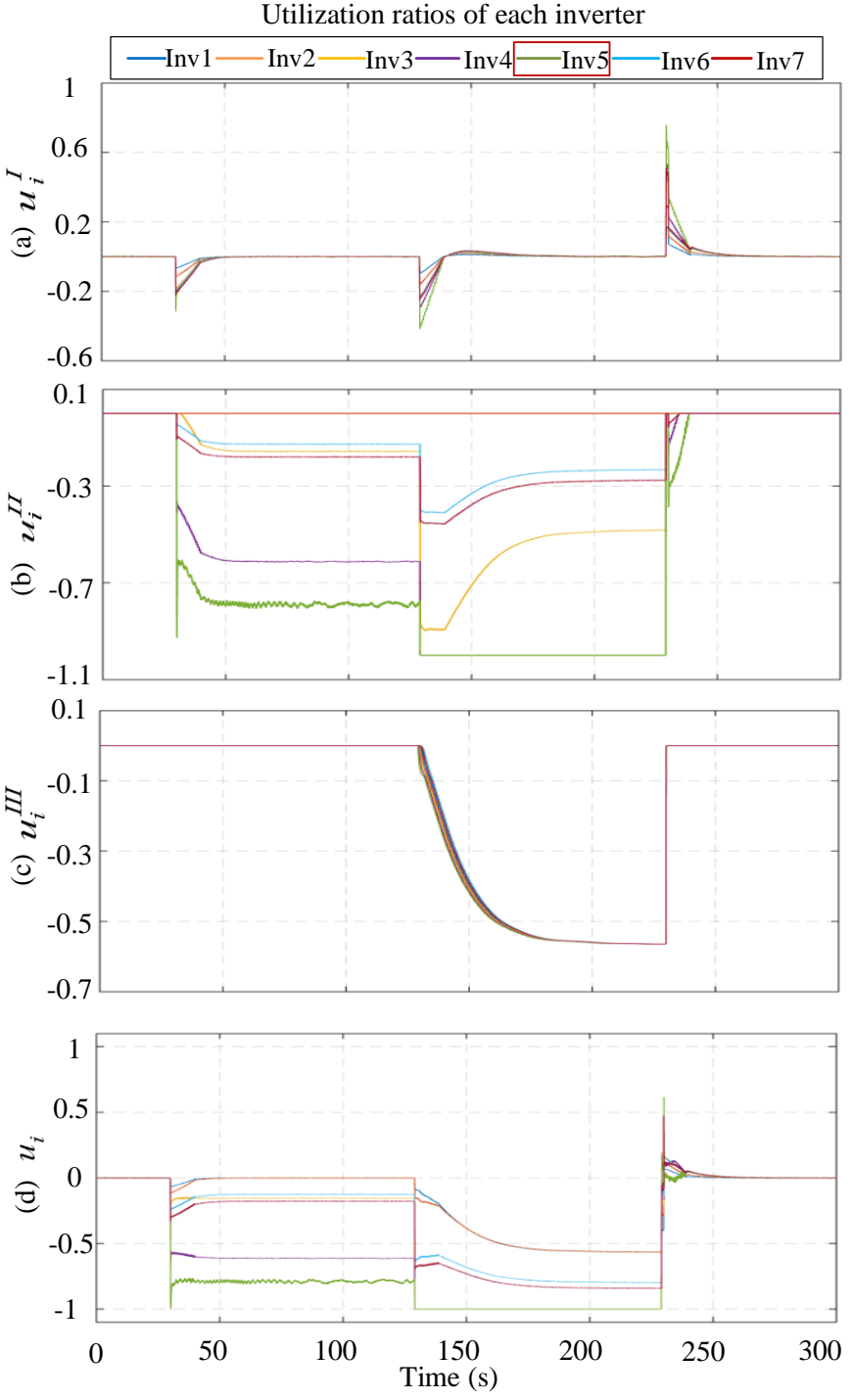

Fig. 8. The utilization ratios of each inverter in Test Case1.

The network voltage profiles, utilization ratios, and the PV inverters reactive power outputs have been shown in Figs. 7-9, respectively. The experimental results are further explained in respective time sequences below:

(i) $0 \mathrm{~s} \leq t<30 \mathrm{~s}$. As the network voltages are in the normal operation range of $[\underset{\sim}{V}, \tilde{V}]$ during this period, the utilization ratios are all zeros and there is no reactive power output from $\mathrm{PV}$ inverters.

(ii) $30 \mathrm{~s} \leq t<130 \mathrm{~s}$. With the increase in load, five of the seven bus voltages are out of $[\underset{\sim}{V}, \tilde{V}]$ but still within $[\underline{V}, \bar{V}]$. The Level II droop control operates in this period to mitigate the voltage deviations locally.

(iii) $130 \mathrm{~s} \leq t<230 \mathrm{~s}$. With the further increase in load, the voltage of bus 5 is out of the range $[\underline{V}, \bar{V}]$, thereby triggering the distributed control. Each inverter generates the same additional utilization ratio for reactive power injection, as shown in Fig. 6 (c).

(iv) $230 \mathrm{~s} \leq t<300 \mathrm{~s}$. With the decrease in load, the network voltages are back to the voltage range of $[0.97,1.03]$. The effect of ramp-rate control mitigates the rate of voltage changes. The integrators of distributed control are reset according to the distributed reset algorithm presented in (13)-(15). All the utilization ratios and reactive power outputs of $\mathrm{PV}$ inverters are then back to zero.

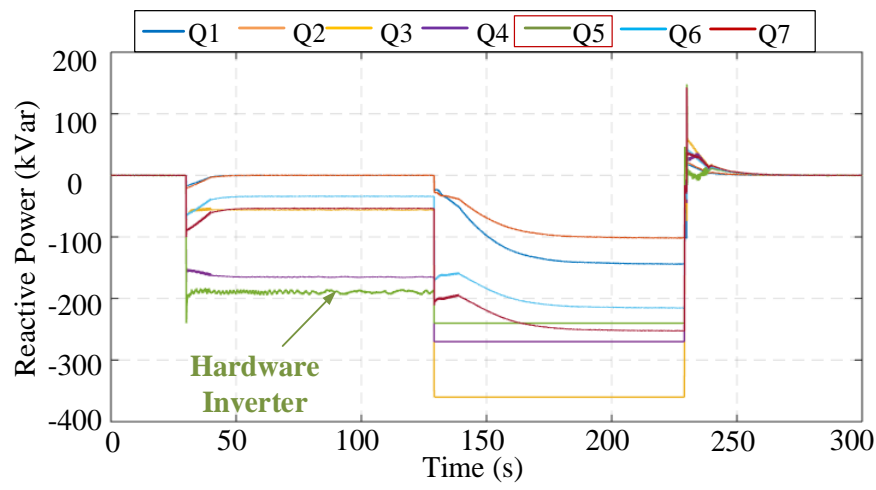

Fig. 9. The reactive power output of PV inverters in Test Case 1.

\section{Test Case 2: Communication Delays}

In Test Case 2, the performance of the proposed control method with communication delays is investigated. The test system conditions remain the same as in Test Case 1. Two types of communication delays are considered: (i) a constant time delay $\tau_{1}(t)=0.5 \mathrm{~s}$, and (ii) a variable time delay representable by Gaussian distribution as:

$$
\tau_{2}(t)=\frac{1}{\sigma \sqrt{2 \pi}} e^{-\frac{1}{2}\left(\frac{x-\mu}{\sigma}\right)^{2}} \mathrm{~s}
$$

where mean value $\mu=0.15$ and variance $\sigma^{2}=0.05$. These two time delays are bounded and satisfy $\tau(t) \leq \bar{\tau}$. The maximum tolerable communication delay can be determined based on the time-delay stability analysis as in [33].

In Fig. 10, the voltage profiles of the hardware PV inverter (bus 5) with the two communication delays are compared. Fig. 11 shows the comparison of utilization ratios $u_{i}^{I I I}(t)$ of each inverter with these two communication delays. As shown in Fig. 10 and 11, the proposed control approach works normally under both constant delay $\tau_{1}(t)$ and variable delay $\tau_{2}(t)$. This validates the effectiveness of the proposed approach under 
different communication delay conditions. It also can be noticed that the increase in communication delay impacts the convergence speed of the distributed control. Therefore, it takes a longer time to control the bus voltage back to 0.95 p.u., as shown in Fig. 10. Furthermore, with the increase in communication delay, the reset time of the integrators also increases, as shown in Fig. 11 (a) and (b).

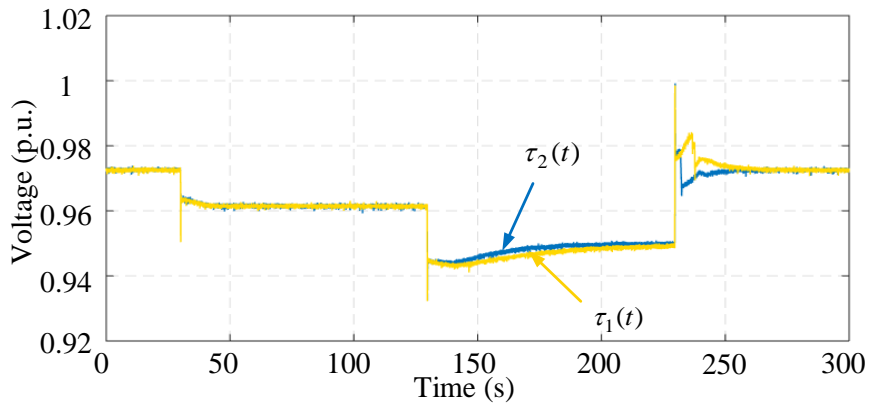

Fig. 10. Voltage profiles of real hardware PV inverter at bus 5 with two types of communication delays.
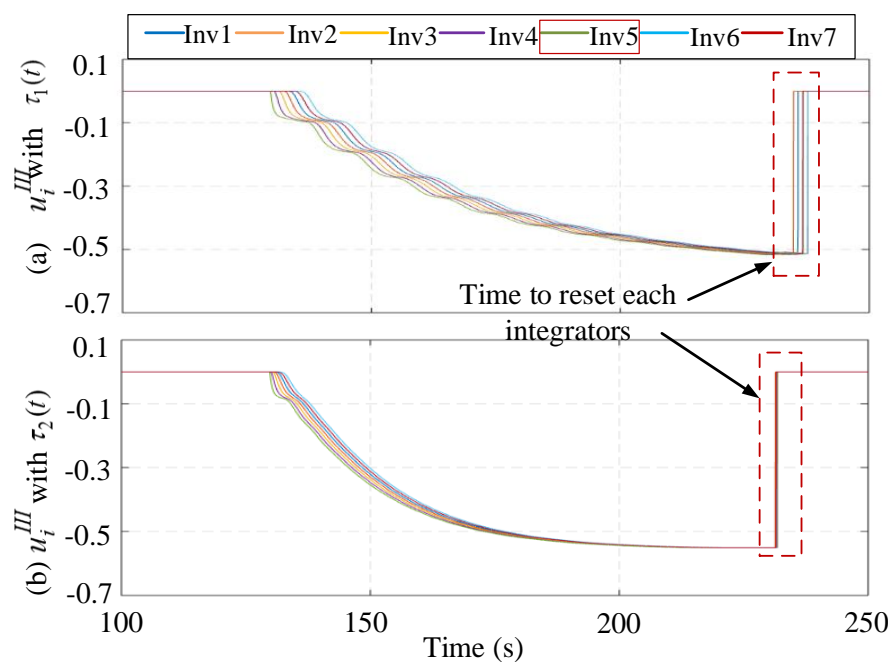

Fig. 11. The comparison of utilization ratios $u_{i}^{I I I}(t)$ with (a) $\tau_{1}(t)$ and (b) $\tau_{2}(t)$.

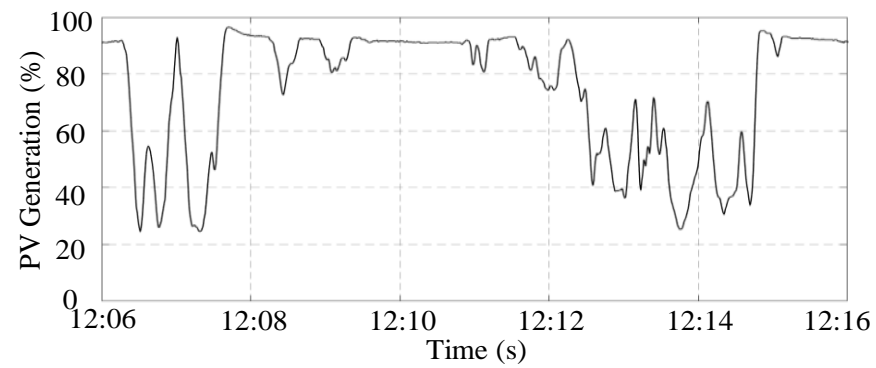

Fig. 12. PV profiles in Test Case 3.

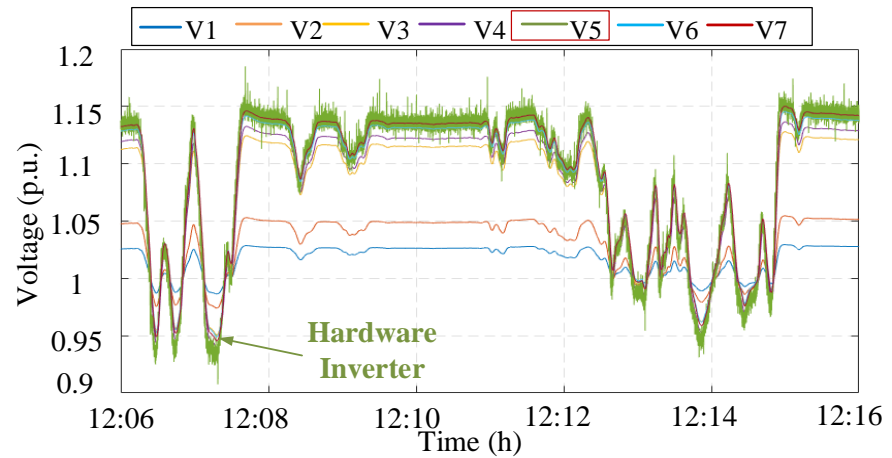

Fig. 13. Voltage profiles without any voltage control.

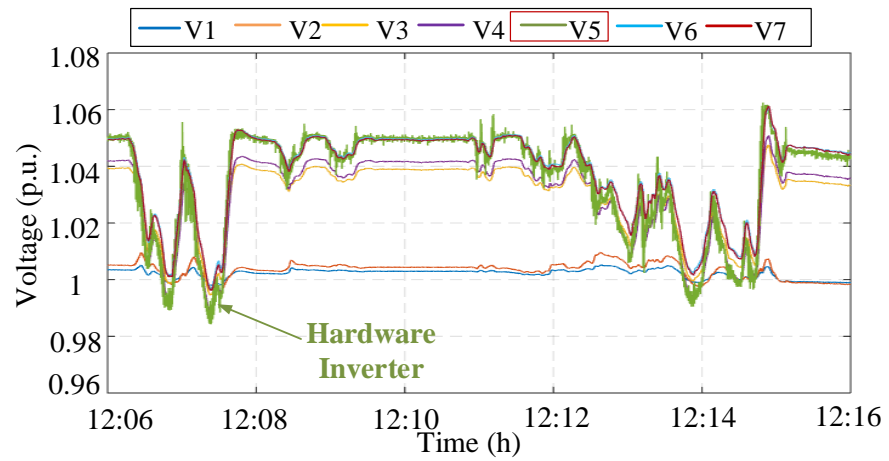

Fig. 14. Voltage profiles with the proposed method in Test Case 3.
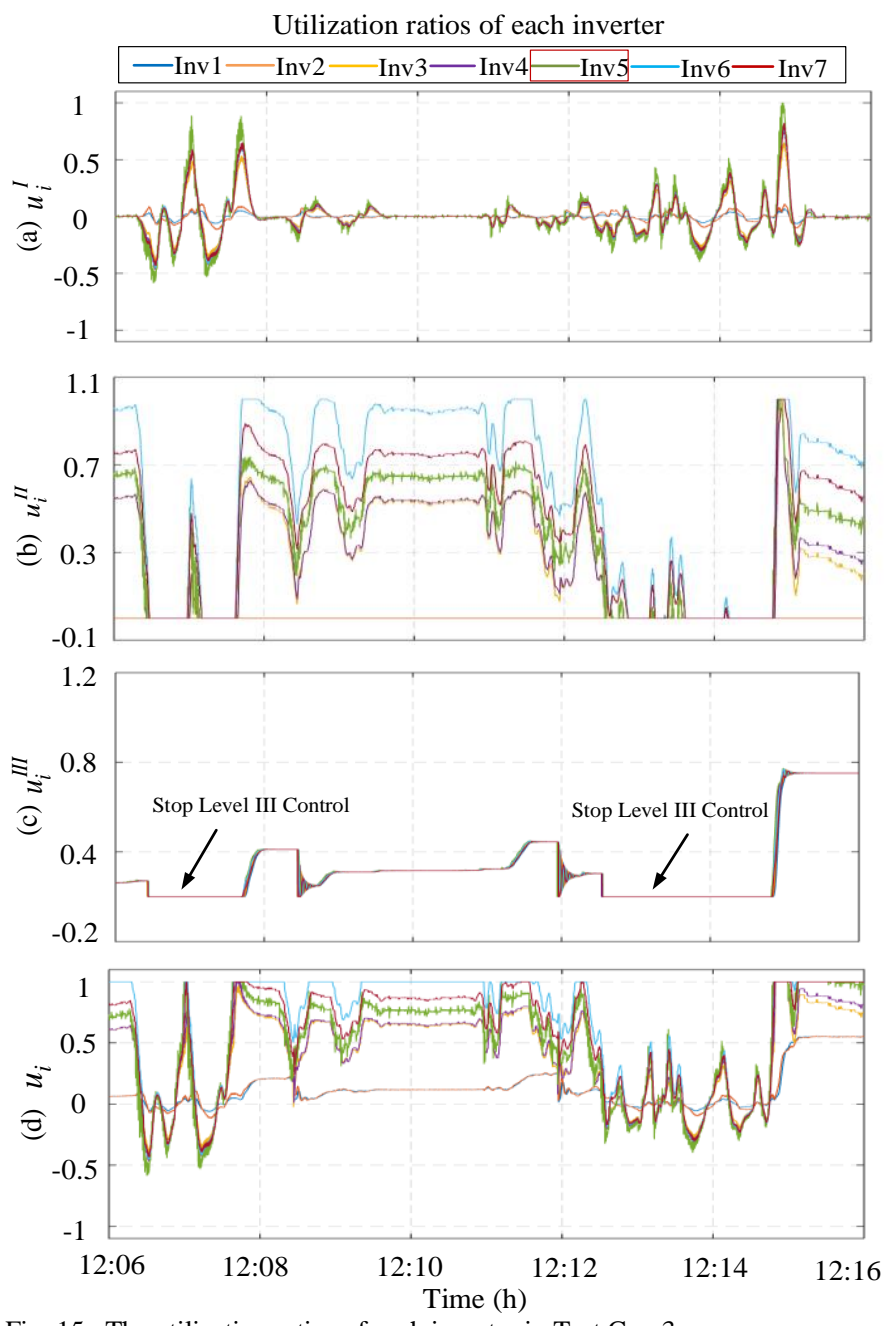

Fig. 15. The utilization ratios of each inverter in Test Case3. 


\section{E. Test Case 3: Voltage Rise with Real PV Data}

In Test Case 3, the ability of the proposed control approach to mitigate voltage rise conditions arising from high PV penetration is tested. Such a situation usually occurs during daytime, when the residential load demand is low while PV generation is at its peak. Ten minutes PV data with one-second resolution as shown in Fig. 12, measured by EPRI in June 2012 [34], is used here. As per the profile shown in Fig. 12, the PV real power output ramps up and down from $20 \%$ to $95 \%$ of its capacity. Therefore, the PV capacity is slightly oversized $\left(\alpha_{i}=0.1\right)$ to enable sufficient reactive power output support even under peak PV generation. The line resistance is increased by ten times (compared to the previous studies, Test Case 1 and 2) to enable emulation of voltage rise issues experienced in a weaker, more resistive distribution grid. The communication delay is considered as $\tau(t)=100 \mathrm{~ms}$. The load percentage is set to $40 \%$ while variations in load are ignored. The voltage profiles without any voltage control are shown in Fig. 13 as a reference case for comparison.

The network voltage profiles, utilization ratios, and reactive power outputs are shown in Figs. 14-16, respectively. As can be observed from Fig. 14, the network voltages can be mostly regulated within $[0.95,1.05]$, except for very short transient periods. The PHIL results reinforce the verification of the proposed control approach to: (i) mitigate voltage rise issues, (ii) mitigate the voltage fluctuations and (iii) work under realworld PV data. In addition, the integrator reset control stops the Level III control when the network voltages are back within safe operational range [0.96, 1.04], as shown in Fig. 14 and Fig. $15(\mathrm{c})$.

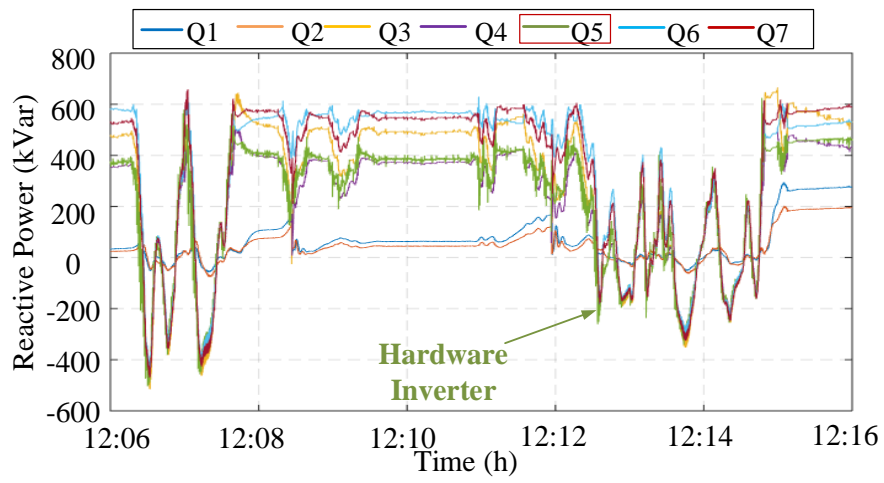

Fig. 16. The reactive power output of each inverter in Test Case 3.

\section{F. Test Case 4: Comparison with Method in [22]}

In Test Case 4, the proposed method is compared with the method in [22] to further demonstrate its advantages. In [22], a two-level voltage var control scheme incorporating a ramp-rate control and distributed leader-follower control is proposed. The distinctive differences with the proposed control, i.e., the droop control and integrator reset, are not considered. The performance of the two methods is compared with repect to the control objective of regulating the voltage within [0.95, 1.05]. The network voltage profiles, utilization ratios, and reactive power outputs with the method in [22] are shown in Figs. 1719 , respectively.

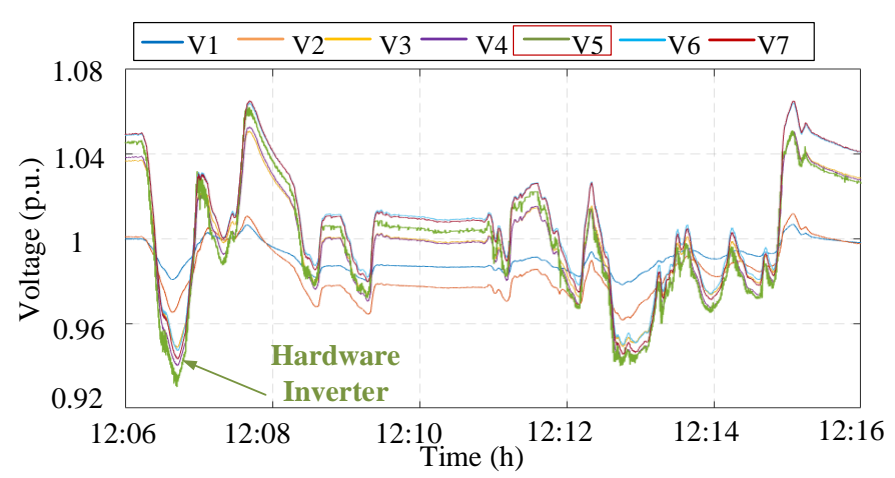

Fig. 17. Voltage profiles in Test Case 4.

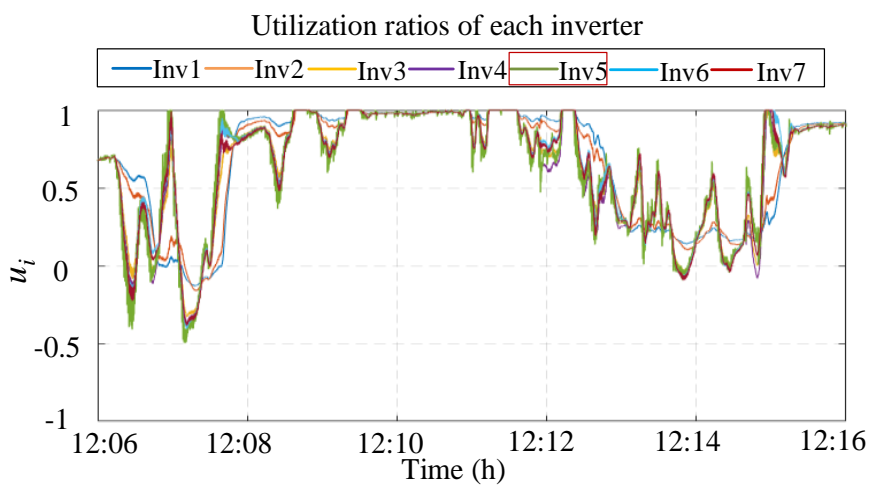

Fig. 18. The utilization ratios of each inverter in Test Case 4.

It can be seen that the method proposed in this paper offers the following two advantages:

(i) The proposed three-level voltage control is fully distributed, while the method in [22] relies on the measurement of voltage at critical buses. Thus the proposed control structure is more resilient than the method in [22], as the critical bus measurement and leader control are not needed.

(ii) Although the method in [22] can also regulate the voltage within $[0.95,1.05]$ except for transient periods in Fig. 17, more reactive power is actually utilized during this process, as compared by Fig. 16 and Fig. 19. The usage of reactive power by the method in [22] is 35\% higher than the proposed method. The utilization ratio of each inverter also indicates more use of reactive power, as compared by Fig. 18 and Fig. 15 (d). The main reason is that the droop control and integrator reset are not considered in [22].

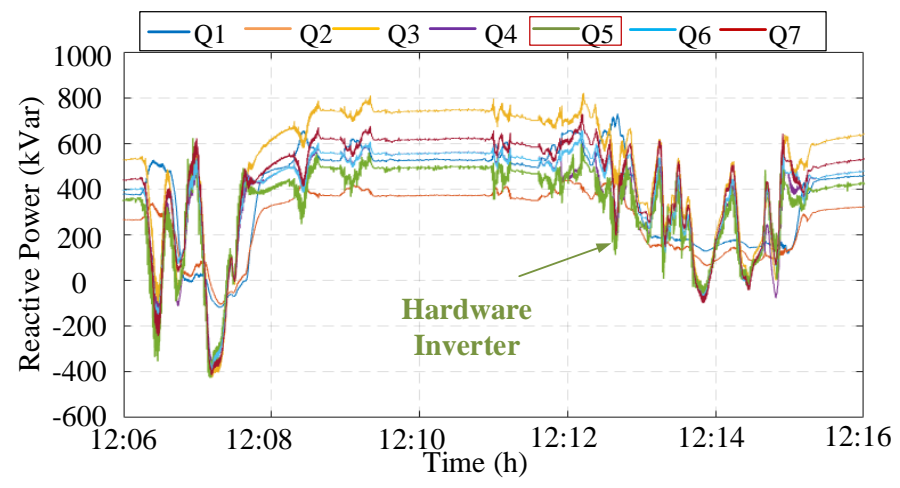

Fig. 19. The reactive power output of each inverter in Test Case 4 . 


\section{CONCLUSION}

In this paper, a three-level coordinated control method for PV inverters has been proposed for voltage regulation in distribution networks. The proposed three-level control can achieve both local voltage control and distributed voltage control by using the reactive power from PV inverters. The stability of the proposed three-level coordinated voltage control is analyzed for different network voltage ranges.

The effectiveness of the proposed control approach has been validated by means of PHIL experiment. The proposed controllers are separately designed and communication links among them are realistically implemented. Compared with most of existing work, this platform allows a more realistic validation of the distributed control method. The results have demonstrated that the three-level control can determine the utilization ratio based on the network voltage conditions. The corresponding reactive power can be provided by each PV inverter to deal with both the voltage fluctuations and voltage limits violation issues. Besides, it has been validated that the proposed control method performs effectively under bounded communication delays. The comparison study with our previous research demonstrates advantages of the proposed three-level voltage control method.

\section{REFERENCES}

[1] Renewables 2018 Global Status Report. [Online]. Available: http://www.ren21.net/wp-content/uploads/2018/06/178652_GSR2018 _FullReport_web_-1.pdf.

[2] F. Ding, Fei, and B. Mather. "On Distributed PV Hosting Capacity Estimation, Sensitivity Study, and Improvement," IEEE Trans. Sustainable Energy, vol. 8, no .3, pp. 1010-1020, Sep. 2016.

[3] R. Tonkoski, D. Turcotte, and T. H. M. El-Fouly, "Impact of high PV penetration on voltage profiles in residential neighborhoods," IEEE Trans. Sustainable Energy, vol. 3, no. 3, pp. 518-527, Jul. 2012.

[4] M. Yilmaz and P. T. Krein, "Review of the impact of vehicle-to-grid technologies on distribution systems and utility interfaces," IEEE Trans. Power Electronics, vol. 28, no. 12, pp. 5673-5689, Nov. 2012.

[5] Pacific Power Engineering Handbook. [Online]. Available: https://www.pacificpower.net/content/dam/pacific_power/doc/Contrac tors_Suppliers/Power_Quality_Standards/1C_5_1.pdf

[6] M. E. Elkhatib, R. El Shatshat, and M. M. A. Salama, "Optimal control of voltage regulators for multiple feeders," IEEE Trans. Power Delivery, vol. 25, no. 4, pp. 2670-2675, Dec. 2009.

[7] M. Chamana, Manohar, and B. H. Chowdhury. "Optimal Voltage Regulation of Distribution Networks With Cascaded Voltage Regulators in the Presence of High PV Penetration," IEEE Trans. Sustainable Energy, vol. 9, no .3, pp.1427-1436, Jul. 2018.

[8] 1547 Series of Interconnection Standards, IEEE SCC21 Standards Coordinating Committee on Fuel Cells, Photovoltaics, Dispersed Generation, and Energy Storage, IEEE Standards Association. [Online].Available: http://grouper.ieee.org/groups/scc21/dr_shared/.

[9] Y. Xu, Z. Y. Dong, R. Zhang, and D. J. Hill, "Multi-timescale coordinated voltage/var control of high renewable-penetrated distribution networks," IEEE Trans. Power Systems, vol.32, no.6, pp. 4398-4408, Nov. 2017.

[10] Y. Wang, et al. "Coordinated control of distributed energy-storage systems for voltage regulation in distribution networks," IEEE Trans. Power Delivery, vol. 31, no .3, pp. 1132-1141, Jun. 2016.

[11] M. J. E. Alam, K. M. Muttaqi, and D. Sutanto, "A multi-mode control strategy for VAr support by solar PV inverters in distribution networks," IEEE Trans. Power Systems, vol. 30, no. 3, pp. 1316-1326, May 2015.

[12] P. Jahangiri and D. C. Aliprantis, "Distributed volt/var control by PV inverters," IEEE Trans. Power Systems, vol. 28, no. 3, pp. 3429-3439, Aug. 2013.

[13] B. A. Robbins, C. N. Hadjicostis, and A. Domínguez-Garcíz, "A twostage distributed architecture for voltage control in power distribution systems," IEEE Trans. Power Systems, vol. 28, no. 2, pp. 1470-1482, May 2013.

[14] R. O. Saber, J. A. Fax, and R. M. Murray, "Consensus and cooperation in networked multi-agent systems," Proceedings of the IEEE, vol. 95, no. 1, pp. 215-233, Jan. 2007.

[15] H. Xin, Z. Qu, J. Seuss, and A. Maknouninejad "A self-organizing strategy for power flow control of photovoltaic generators in a distribution network," IEEE Trans. Power Systems, vol. 26, no. 3, pp. 1462-1473, Aug. 2011.

[16] G. Mokhtari, A. Ghosh, G. Nourbakhsh, and G. Ledwich, "Smart robust resources control in LV network to deal with voltage rise issue," IEEE Trans. Sustainable Energy, vol. 4, no. 4, pp. 1043-1050, Oct. 2013.

[17] M. Andreasson, D. V. Dimarogonas, H. Sandberg, and K. H. Johansson, "Distributed PI-control with applications to power systems frequency control," American Control Conference (ACC), 2014.

[18] J. Simpson-Porco, et al. "Secondary frequency and voltage control of islanded microgrids via distributed averaging," IEEE Trans. Industrial Electronics, vol. 62, no.11, pp. 7025-7038, Nov. 2015.

[19] Y. Wang, et. al. "Cyber-Physical Design and Implementation of Distributed Event-Triggered Secondary Control in Islanded Microgrids," IEEE Trans. Industry Applications, vol. 55, no. 6, pp. 5631-5642, Nov.-Dec. 2019.

[20] Y. Zheng, D. Hill, K. Meng, S. Hui, "Critical bus voltage support in distribution systems with electric springs and responsibility sharing," IEEE Trans. Power Systems, vol. 32, no. 5, pp. 3584-93, Sep. 2017.

[21] H. Bidgoli, and T. V. Cutsem, "Combined local and centralized voltage control in active distribution networks," IEEE Trans. Power Systems, vol. 33, no .2, pp. 1374-1384, 2018.

[22] Y. Wang, et al. "Decentralised-distributed hybrid voltage regulation of power distribution networks based on power inverters," IET Gen., Trans. \& Dist., vol. 13, no .3, pp. 444-451, 2018.

[23] H. Zhu, and H. J. Liu. "Fast local voltage control under limited reactive power: Optimality and stability analysis," IEEE Trans. Power Systems, vol. 31, no .5, pp. 3794-3803, Sep. 2016.

[24] S. Alyami, Y. Wang, C. Wang, J. Zhao and B. Zhao, "Adaptive real power capping method for fair overvoltage regulation of distribution networks with high penetration of PV systems," IEEE Trans. Smart Grid, vol. 5, no. 6, pp. 2729-2738, Nov. 2014.

[25] Y. Zhang, Y. Xu, H.M. Yang, and Z.Y. Dong, "Voltage RegulationOriented Co-Planning of Distributed Generators and Battery Storage in Distribution Networks," Int. J. Electrical Power and Energy Systems, vol. 105, pp. 79-88, Feb. 2019.

[26] M. J. E. Alam, K. M. Muttaqi, and D. Sutanto, "A novel approach for ramp-rate control of solar PV using energy storage to mitigate output fluctuations caused by cloud passing," IEEE Trans. Energy Conversion, vol. 29, no .2, pp. 507-518, Jun. 2014.

[27] Y. Li, and C. N. Kao, "An accurate power control strategy for powerelectronics-interfaced distributed generation units operating in a lowvoltage multibus microgrid," IEEE Trans. Power Electronics, vol. 24, no .12, pp. 2977-2988, Dec. 2009.

[28] K. Baker, et al. "Network-cognizant voltage droop control for distribution grids," IEEE Trans. Power Systems, vol. 33, no .2, pp. 2098-2108, Mar. 2018.

[29] C. Zhang, Y. Xu, Z.Y. Dong, and J. Ravishankar "Three-Stage Robust Inverter-Based Voltage/Var Control for Distribution Networks with High PV," IEEE Trans. Smart Grid, vol.10, no.1, pp. 782-793, Jan. 2019.

[30] R. Tonkoski, et al, "Coordinated active power curtailment of grid connected PV inverters for overvoltage prevention," IEEE Trans. Sustainable Energy, vol. 2, no. 2, pp. 139- 147, Apr. 2011.

[31] P. C. Kotsampopoulos, et al., "The Limitations of Digital Simulation and the Advantages of PHIL Testing in Studying Distributed Generation Provision of Ancillary Services," IEEE Trans. Industrial Electronics, vol. 62, no. 9, pp. 5502-5515, Sept. 2015.

[32] E. Guillo-Sansano, A. J. Roscoe and G. M. Burt, "Harmonic-byharmonic time delay compensation method for PHIL simulation of low impedance power systems," 2015 International Symposium on Smart Electric Distribution Systems and Technologies (EDST), Vienna, 2015, pp. 560-565.

[33] J. Rocabert, et al. "Control of power converters in AC microgrids," IEEE Trans. Power Electronics, vol. 27, no .11, pp. 4734-4749, Nov. 2012.

[34] C. Dong, et. al. "Time-delay stability analysis for hybrid energy storage system with hierarchical control in DC microgrids," IEEE Trans. Smart Grid, 2017. 
[35] 1-second resolution data, EPRI. [Online]. Available: http://dpv.epri.com/measurement_data.html.

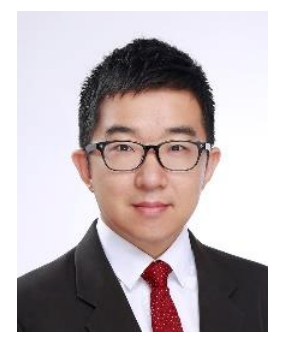

Yu Wang (S'12-M'17) received the B.Eng. degree from Wuhan University, Wuhan China in 2011, and the M.Sc. and Ph.D. degree from Nanyang Technological University, Singapore in 2012 and 2017 , respectively. He is currently a research fellow in Nanyang Technological University, Singapore. His research interests include distributed control and optimization, energy storage systems, microgrids and smart grids.

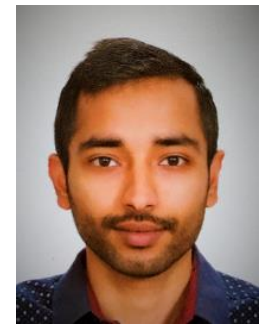

Mazheruddin H. Syed (GS'11-M'18) was born in Hyderabad, India. He received his B.E. degree in electrical and electronics engineering from Osmania University, India, in 2011, his M.Sc. degree in electrical power engineering from Masdar Institute of Science and Technology, UAE, in 2013, and his Ph.D. degree in electrical power systems from the University of Strathlcyde, Glasgow, Scotland, in 2018. Currently, he is a Research Associate at the Institute for Energy and Environment, University of Strathclyde, Glasgow, Scotland. His research interests include demand side management, distributed control, controller and power hardware in the loop simulations, and systems level validations.

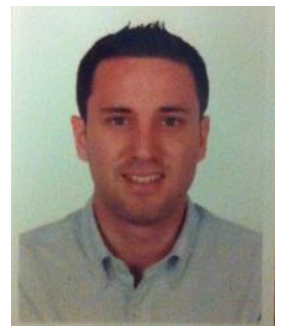

Efren Guillo-Sansano (GS'13-M'18) received his M.Sc. and Ph.D. degrees in Electrical Power Engineering from the University of Strathclyde, Glasgow, Scotland, in 2013 and 2018. Currently, he is a Research Associate with the Institute for Energy and Environment at the University of Strathclyde. His research interests include the testing and validation of distributed energy resources and power electronics, power hardware in the loop, distributed control, and ancillary services.

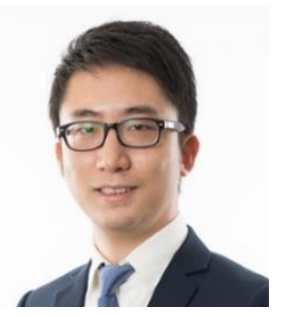

Yan Xu (S'10-M'13) received the B.E. and M.E degrees from South China University of Technology, Guangzhou, China in 2008 and 2011, respectively, and the Ph.D. degree from The University of Newcastle, Australia, in 2013. He is now the Nanyang Assistant Professor at School of Electrical and Electronic Engineering, Nanyang Technological University (NTU), and a Cluster Director at Energy Research Institute @ NTU (ERI@N), Singapore. Previously, he held The University of Sydney Postdoctoral Fellowship in Australia. His research interests include power system stability and control, microgrid, and dataanalytics for smart grid applications. $\mathrm{Dr} \mathrm{Xu}$ is an Editor for IEEE TRANSACTIONS ON SMART GRID, CSEE Journal of Power and Energy Systems, and an Associate Editor for IET Generation, Transmission \& Distribution.

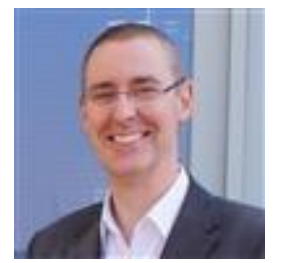

Graeme M. Burt (M'95) received the B.Eng. degree in electrical and electronic engineering, and the Ph.D. degree in fault diagnostics in power system networks from the University of Strathclyde, Glasgow, U.K., in 1988 and 1992, respectively. He is currently a Professor of electrical power systems at the University of Strathclyde where he co-directs the Institute for Energy and Environment, directs the Rolls-Royce University Technology Centre in Electrical Power Systems, and is lead academic for the Power Networks Demonstration Centre (PNDC). In addition, he serves as spokesperson for the board of DERlab e.V., the association of distributed energy laboratories. His research interests include the areas of power system protection and control, distributed energy, and experimental validation. 